\title{
THERMOELECTRIC POWER OF STRONTIUM UP TO 6.5 GPa
}

\section{Divakar, M. Mohan and A.K. Singh}

Materials Science Division, National Aeronautical Laboratory, Bangalore 560 017, India

(Received 15 October 1981 by M. Cardona)

The thermoelectric power of Sr has been measured up to $6.5 \mathrm{GPa}$ using an opposed anvil set up. The results have been compared with those for $\mathrm{Yb}$.

THE PRESSURE-TEMPERATURE PHASE diagrams of $\mathrm{Yb}$ and $\mathrm{Sr}$ exhibit striking similarities [1-4]. The electrical resistance at room temperature increases with pressure in both cases and drops at 4.0 GPa in case of $\mathrm{Yb}$ and $3.6 \mathrm{GPa}$ in case of $\mathrm{Sr}[5]$. This drop in electrical resistance is associated with f.c.c. $+\mathbf{b} . \mathbf{c} . c_{\text {. }}$ transformation $[6,7]$. We have measured at room temperature, the thermoelectric power (TEP) of $\mathrm{Sr}$ up to $6.5 \mathrm{GPa}$. In this communication we report the results of these measurenents and compare them with those of $\mathrm{Yb}$ reported arlier $[8,9]$.

The TEP as a function of pressure was measured ssing a tungsten carbide opposed anvil (12 $\mathrm{mm}$ flat face) et up with pyrophyllite gasket and either talc or epoxy $s$ pressure transmitting medium. A temperature gradient vas established across the sample by heating one end of he sample. The temperature and the potential differen:es between two points on the sample were measured vith chromel-alumel thermocouples and K-5 (Leeds Vorthup) nanovolt potentiometer. The pressure on the ample was determined by calibrating the ram pressure ssing $\mathrm{B}_{\mathrm{I}-\mathrm{II}}(2.55 \mathrm{GPa}), \mathrm{Tl}_{\mathrm{II}-\mathrm{III}}$ and $\mathrm{Bi}_{\mathrm{II}-\mathrm{IV}}(7.6 \mathrm{GPa})$ ransitions. The details of the experimental arrangement Ire published elsewhere [9]. The Sr sample used in the Jesent measurements was $99.5 \%$ pure from Research Jrganic/Inorganic Co., U.S.A: A small piece of $\mathrm{Sr}$ was ressed between two flat surfaces to give a $0.1 \mathrm{~mm}$ thick iheet. The specimens measuring nearly $0.1 \times 0.5 \times \mathrm{Smm}$ Nere cut from this sheet. During the entire operation the ample surface were covered with machine oil. Finally, he specimens were scraped clean and loaded under argon atmosphere in the cell. The specimen pressure was increased in steps of $0.1 \mathrm{GPa}$. After each increase in pressure $5 \mathrm{mt}$ were allowed for the cell to stabilise, and then the measurements were made. The f.c.c.-b.c.c. transition was sluggish and it was found necessary to allow for $15 \mathrm{mt}$ waiting time.

A typical plot of TEP of $\mathrm{Sr}$ against pressure is shown in Fig, 1. It is seen that TEP increases with the increase in pressure and reaches a maximum at $3.6 \mathrm{GPa}$. The subsequent drop in TEP is associated with the f.c.c.-b.c.c. transition. In the b.c.c. phase TEP decreases

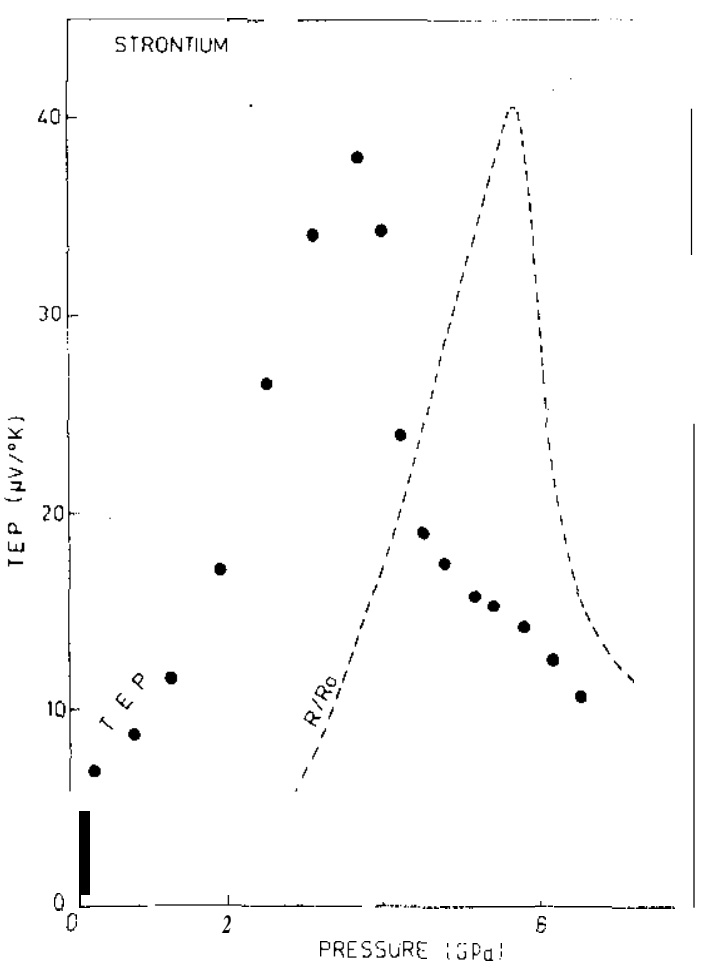

Fig. I. Thermoelectric power and resistivity of strontium as a function of pressure. For clarity, pressure scale for resistivity is shifted to the right by $2 \mathrm{GPa}$.

smoothly. For a ready reference the electrical resistivity measured on the same batch of specimens is also plotted as a function of pressure.

A simple consideration shows that a crystal composed of divalent atoms is insulating at large interatomic distances with filled s-band and empty p-band [10]. With a decrease in interatomic distance such a crystal can become a metal or a semiconductor. A detailed band structure calculation for f.c.c. calcium differs from the simple model in that there exists a line of degeneracy between the first and the second band [11]. This model predicts that a divalent f.c.c. metal will become under pressure a semi-metal but not a semiconductor. However, if the spin-orbit coupling is strong it can remove the band degeneracy. The Fermi 


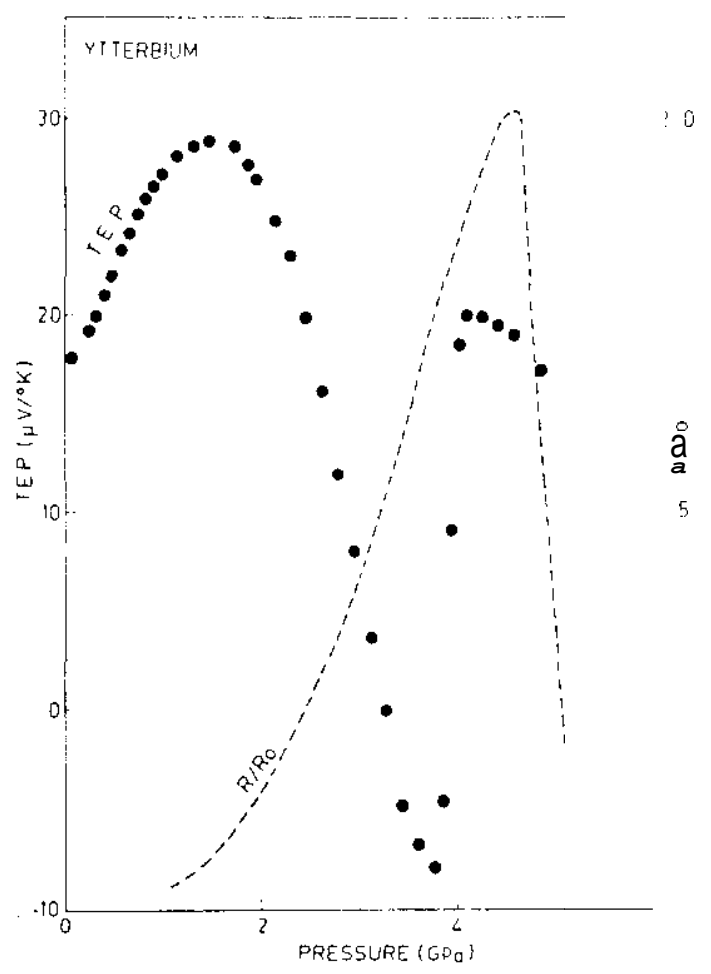

Fig. 2. Thermoelectric power and resistivity of ytterbium as a function of pressure. For clarity, pre. sure scale for resistivity is shifted to the right by $1 \mathrm{GPa}$.

surface in such a case can shrink to zero under pressure and a semiconducting state can result. The resistivity data under pressure suggest that $\mathrm{Sr}$ remains a semimetal up to $3.6 \mathrm{GPa}$ before undergoing f.c.c.-b.c.c. transition $[1,12]$. On the other hand $\mathrm{Yb}$, a semimetal, becomes semiconductor near $1.3 \mathrm{GPa}$ remains a semiconductor before undergoing f.c.c.-b.c.c. transition at 4.0 GPa. This difference in the behaviour is expected because of large spin-orbit coupling in $\mathrm{Yb}$.

The TEP and the resistivity of $\mathrm{Yb}$ are plotted in Fig. 2 as a function of pressure. It is seen that for both
$\mathrm{Sr}$ and $\mathrm{Yb}, \mathrm{TEP}$ is large positive at one atmosphere. The sign of TEP agrees in both cases with the sign of the Hall co-efficient [13]. The positive sign of the Hall coefficient has been interpreted in the frame work of two band model as arising from the fact that the mobility of the holes is larger than that of the electron [8]. In the initial stages, where both $\mathrm{Yb}$ and $\mathrm{Sr}$ are semi. metals, the TEP increases with pressure because of a gradual removal of band overlap. In the case of $\mathrm{Sr}$ the increase in TEP with an increase in pressure continues until the f.c.c.-b.c.c. transformation pressure is reached. This is expected as $\mathrm{Sr}$ remains a semimetal up to the f.c.f.-b.c.c. transformation pressure. In the semiconducting region TEP of $\mathrm{Yb}$ decreases with the increase in pressure.

\section{REFERENCES}

1. P.C. Souers \& G. Jura, Science 140,481 (1963).

2. A. Jayaraman, W. Kletnent J.s. \& G.C., Kennedy, Phys. Rev. 132,1620 (1963).

3. A. Jayaraman, Phys. Rev. 135, A1065 (1964).

4. D.R. Stephens, J. Phys. Chem. Solids 26,943 (1965).

5. P.W. Brideman. Collected Experimental Papers, Harvatd Univ. Press, Cambridge, Mass. (1964).

6. D.B. McWhar \& A. Jararaman, Appl. Phys. Lett. 3, 129, (1963).

7. H.T. Hall. J.D. Barnett \& L. Merrill, Science 139, 111 (1963).

8. T.G. Ramesh, V. Shubha \& S. Ramaseshan, J. Phys. F: Metal Phys. 7,981 (1977).

9. A.K. Singh \& Geetha Ramani, Rev. Sci. Instrum. 49, 1324 (1978).

10. N.F. Mott \& H. Jones, Theory of the Properties of Metals and Alloys. Oxford University Press, New York (1936).

11. B. Basavari, A.O.E. Animalu \&. V. Heine, Phys. Rev. 154,535 (1967).

12. D.B. McWhan, T.M. Rice\& P.H. Schmidt, Phys. Rev. 177, 1063 (1969).

13. W.B. Halzapfel \& D. Severin, Phys. Lett. 34A, 371 (1971). 